\title{
Radiation and Diffusion Thermo Effects of Visco-Elastic Fluid Past a Porous Surface in the Presence of Magnetic Field and Chemical Reaction with Heat Source
}

\author{
B. Mallikarjuna Reddy ${ }^{1 *}$, D. Chenna Kesavaiah ${ }^{2}$, G. V. Ramana Reddy ${ }^{3}$ \\ ${ }^{1}$ Research scholar, Koneru Lakshmaiah Deemed to be University \\ Vaddeswaram, Guntur, AP, India \\ ${ }^{2}$ Department of Humanities \& Science, K G Reddy College of Engineering \& Technology \\ Chilkur (V), Moinabad (M), R R Dist, TS - 501504, India \\ ${ }^{3}$ Department of Mathematics, Koneru Lakshmaiah Deemed to be University \\ Vaddeswaram, Guntur, AP, India \\ *Corresponding author's email: mallikb2010 [AT] gmail.com
}

\begin{abstract}
An analysis of two dimensional free convective hydro-magnetic flow of an electrically conducting visco-elastic fluid past a vertical porous surface has been studied in the presence of magnetic field and chemical reaction of first order with heat source. Simultaneously effects of heat and mass transfer are also studied in combination of the physics of diffusion thermo (Dufour effect) and suction at the plate is assumed to be constant. The equations governing are solved by perturbation technique. Analytical expressions for velocity, temperature and concentration fields, non-dimensional skin friction coefficient and Nusselt number are obtained. The results are analyzed graphically for various values of visco-elastic parameter along with a combination of other flow parameters involved in the solution.
\end{abstract}

Keywords--- Radiation, Viscous drag, Hydro magnetic Flow, Walter's liquid (Model B ') and Heat source

\section{INTRODUCTION}

The problem of flow, heat and mass transfer on mixed convection over a stretching surface in a fluid-saturated porous medium could be very practicable in numerous applications in the polymer technology and metallurgy. For instance, it occurs in many metallurgical processes which involve the cooling of con- tenuous strips or filaments by drawing them through a quiescent fluid and that in the process of drawing, these strips are some- times stretched. The properties of the final products depend greatly on the rate of cooling so that final products of desired characteristics might be achieved. The rate of cooling can be controlled by drawing such strips through an electrically con- ducting fluid in the presence of transverse magnetic field. Heat transfer is the study of the flow of heat. It is an energy transfer due to temperature difference in a medium or between two or more media. In chemical engineering, to predict rates of heat transfer in a variety of process situations. In accident scenarios involving fire and the transport of explosive material, the time available for escape is dependent on the heat transfer rate from the fire to the energetic material. Dulal Pal and Hiranmoy Mondal [2] Soret and Dufour effects on MHD non-Darcian mixed convection heat and mass transfer over a stretching sheet with non-uniform heat source/sink. Rita Choudhury and Sajal Kumar Das [18] Viscoelastic MHD fluid flow over a vertical plate with Dufour and Soret effects, Chen [1] an analytic solution of MHD flow and heat transfer for two types of viscoelastic fluid over a stretching sheet with energy dissipation, internal heat source and thermal radiation. Dulal Pal and Sewli Chatterjee [3] MHD mixed convective heat and mass transfer in a viscoelastic fluid in a porous medium towards a stretching sheet with viscous-Ohmic heating and chemical reaction. Rafael Cortell Bataller [17] Effects of heat source/sink, radiation and work done by deformation on flow and heat transfer of a visco-elastic fluid over a stretching sheet.

From technological point of view, the visco-elastic MHD convection flow problems are very important in both theoretical and experimental studies as they have overwhelming implications in various fields as petroleum industries, cooling of nuclear reactors, boundary layer control in aerodynamics, crystal growth ect. Diffusion rates can be changed tremendously with chemical reactions. A few representative areas of interest in which heat and mass transfer combined along with the chemical reaction play significant role in chemical industries like in food processing and polymer production. The idea of first order chemical reaction where the rate of reaction is directly proportional to the 
concentration itself has been analyzed by Rajagopal [7, 8] and Rajagopal and Gupta [6]. On the other hand, Abel and Veena [20] investigated a viscoelastic fluid flow and heat transfer in a porous medium over a stretching sheet and observed that the dimensionless surface temperature profiles increases with an increase in viscoelastic parameter $(k)$; however, later, Abel et al. [19] studied the effect of heat transfer on MHD viscoelastic fluid over a stretching surface and an important finding was that the effect of viscoelasticity is to decrease the dimensionless surface temperature profiles in that flow. Furthermore, Char [11] studied MHD flow of a viscoelastic fluid over a stretching sheet; however, only the thermal diffusion is considered in the energy equation. Later, Sarma and Rao [12], Vajravelu and Roper [5] and Cortell [16] analyzed the effects of work due to deformation in that equation. Another effect which bears great importance on heat transfer is the viscous dissipation. When the viscosity of the fluid and or the velocity gradient is high, the dissipation term becomes important. Consequently, the effects of viscous dissipation are also included in the energy equation; Ch Kesavaiah [22] studied Effects of the chemical reaction and radiation absorption on an unsteady MHD convective heat and mass transfer flow past a semi-infinite vertical permeable moving plate embedded in a porous medium with heat source and suction. Chenna Kesavaiah et.al [21] Natural convection heat transfer oscillatory flow of an elastico-viscous fluid from vertical plate, Bhavana and Chenna Kesavaiah [23] Perturbation solution for thermal diffusion and chemical reaction effects on MHD flow in vertical surface with heat generation, Bhavana et.al [24] The Soret effect on free convective unsteady MHD flow over a vertical plate with heat source, Mallikarjuna Reddy et.al. [26] Studied effects of radiation and thermal diffusion on MHD heat transfer flow of a dusty viscoelastic fluid between two moving parallel plates.

The study of MHD flow of viscoelastic fluid over a continuously moving surface has a wide range of application in the production of synthetic sheet, of plastic sheet, cooling of metallic sheet, aerodynamic extrusion etc. in view of the above some of the authors studied by Andersson [4] examined the flow of viscoelastic fluid over a stretching sheet under the influence of uniform magnetic field. Abel et al.[10] studied convective heat and mass transfer in a viscoelastic fluid flow through a porous medium over a stretching sheet. Makinde [13] analysed hydromagnetic boundary layer flow and mass transfer past a vertical plate in a porous medium with constant heat flux. Computational dynamics of hydromagnetic stagnation-point flow towards a stretching sheet was studied by Makinde and Charles [14]. Sibanda and Makinde [15] described MHD flow and heat transfer due to a rotating disk in a porous medium with Ohmic heating and viscous dissipation, Srinathuni Lavanya et.al. [25] Radiation, heat and mass transfer effects on magnetohydrodynamic unsteady free convective Walter's memory flow past a vertical plate with chemical reaction through a porous medium.

The aim of the present study is to investigate radiation and diffusion - thermo (Dufour number) effects on MHD free convective heat and mass transfer flow of a viscoelastic fluid past an infinite vertical plate with homogeneous chemical reaction of first order. The solutions for velocity, temperature and concentration fields are obtained by using relevant boundary conditions. The viscoelastic fluid flow is characterized by Walters's liquid (Model B).

The constitutive equation for Walter's liquid (Model B) is

$\sigma_{i k}=-p g_{i k}+\sigma_{i k}^{\prime}$

$\sigma^{\prime i k}=2 \eta_{0} e^{i k}-2 k_{0} e^{i k}$

where $\sigma_{i k}$ is the stress tensor, $\rho$ is isotropic pressure, $g_{i k}$ is the metric tensor of a fixed co-ordinate system $x_{i}, v_{i}$ is the velocity vector, the contravarient form of $e^{i k}$ is given by

$e^{\prime i k}=\frac{\partial e^{i k}}{\partial t}+V^{m} e_{m}^{i k}-v_{m}^{k} e^{i m}-v_{m}^{i} e^{m k}$

It is the convicted derivative of the deformation rate tensor $e^{i k}$ defined by

$2 e^{i k}=v_{i, k}+v_{k, i}$

Here $\eta_{0}$ is the limiting viscosity at the small rate of shear which is given by

$\eta_{0}=\int_{0}^{\infty} N(\tau) d \tau$ and $k_{0}=\int_{0}^{\infty} \tau N(\tau) d \tau$

$N(\tau)$ being the relaxation spectrum. This idealized model is a valid approximation of Walter's liquid (Model B) taking very short memories into account so that terms involving

$\int_{0}^{\infty} t^{n} N(\tau) d \tau \quad n \geq 2$ have been neglected. 


\section{FORMULATION OF THE PROBLEM}

A study of two dimensional free convective electrically conducting flow of visco-elastic fluid past a vertical porous plate has been investigated in presence of radiation and constant suction. The magnetic field of uniform strength $B_{0}$ is applied in the transverse direction to the plate. In this paper, we have studied the simultaneous heat and mass transfer mechanisms of governing fluid flow in connection with chemical reaction and diffusion thermo. Let $x$-axis be taken along the plate in the vertically upward direction and $y$-axis be taken along the normal to the plate. Then neglecting the induced magnetic field and using Boussinesq approximation, the governing equations of motion are as follows:

$$
\begin{aligned}
& \frac{\partial v^{\prime}}{\partial y^{\prime}}=0 \\
& \rho v^{\prime} \frac{\partial u^{\prime}}{\partial y^{\prime}}=\rho g \beta\left(T^{\prime}-T_{\infty}^{\prime}\right)+\rho g \beta^{*}\left(C^{\prime}-C_{\infty}^{\prime}\right)+\eta_{0} \frac{\partial^{2} u^{\prime}}{\partial y^{\prime 2}}-k_{0}\left(v^{\prime} \frac{\partial^{3} u^{\prime}}{\partial y^{\prime 3}}\right)-\sigma B_{0}^{2} u \\
& \rho C_{p}\left(v^{\prime} \frac{\partial T^{\prime}}{\partial y^{\prime}}\right)=k_{1}\left(\frac{\partial^{2} T^{\prime}}{\partial y^{\prime 2}}\right)-\frac{\partial q_{r}}{\partial y^{\prime}}-Q_{0}\left(T-T_{\infty}\right)+\left(\rho \frac{D_{m} K_{T}}{C_{s}} \frac{\partial^{2} C^{\prime}}{\partial y^{\prime 2}}\right) \\
& v^{\prime} \frac{\partial C^{\prime}}{\partial y^{\prime}}=D\left(\frac{\partial^{2} C}{\partial y^{2}}\right)-K r^{\prime}\left(C^{\prime}-C_{\infty}^{\prime}\right)
\end{aligned}
$$

The relevant boundary conditions are

$$
\begin{array}{lrl}
u^{\prime}=U, v^{\prime}=-v_{0}, T^{\prime} & =T_{w}^{\prime}, C=C_{\infty} & y^{\prime}=0 \\
u \rightarrow 0, & T^{\prime} \rightarrow T_{\infty}^{\prime}, C \rightarrow C_{\infty}^{\prime} & y \rightarrow \infty
\end{array}
$$

Where $u$ and $v$ are the velocity components along and perpendicular to the surface, $g$ is the acceleration due to gravity, $T$ the temperature of the fluid near the plate, $T_{\infty}$ the free stream temperature, $C$ concentration, $\beta$ the coefficient of thermal expansion, $\beta^{*}$ is the volumetric coefficient of expansion of the spices concentration, $k$ the thermal conductivity, $C_{p}$ the specific heat of constant pressure, $B_{0}$ the magnetic field coefficient, $\mu$ viscosity of the fluid, $\rho$ the density, $\sigma$ the magnetic permeability of fluid $V_{0}$ constant suction velocity, $v$ the kinematic viscosity and $D$ chemical molecular diffusitivity.

We introduce the following non-dimensional quantities

$$
\begin{aligned}
& u=\frac{u^{\prime}}{U}, y=\frac{y^{\prime} U}{v}, M=\frac{\sigma B_{0}{ }^{2} v}{\rho U^{2}}, \theta=\frac{\left(T^{\prime}-T_{\infty}^{\prime}\right)}{T_{w}^{\prime}-T_{\infty}^{\prime}}, \operatorname{Pr}=\frac{\eta_{0} C_{p}}{k_{1}}, \lambda=\frac{v_{0}}{U} \\
& C=\frac{\left(C^{\prime}-C_{\infty}^{\prime}\right)}{C_{w}^{\prime}-C_{\infty}^{\prime}}, G r=\frac{\rho \beta g v^{2}\left(T_{w}^{\prime}-T_{\infty}^{\prime}\right)}{U^{3}}, \quad G m=\frac{\rho \beta^{*} g\left(C_{w}^{\prime}-C_{\infty}^{\prime}\right)}{U^{3}} \\
& S c=\frac{v}{D}, K r=\frac{K r^{\prime} v}{U^{2}}, Q_{0}=\frac{\phi U^{2} \rho C_{p}}{v}, R=\frac{16 a^{*} v^{2} \sigma T_{\infty}^{\prime 3}}{k_{1} U^{2}}, k=\frac{k_{0} U^{2}}{\rho v^{2}}
\end{aligned}
$$

Where $G r$ is Grashof number, $\operatorname{Pr}$ is Prandtl number, $M$ is Magnetic number, $S c$ is Schmidt number, $K r$ is Chemical reaction parameter, $\phi$ is heat source parameter, $k$ is dimensionless visco-elastic parameter, $D u$ is the coefficient of mass diffusivity and $R$ is radiation parameter.

The non-dimensional forms of the equations (2) to (4) are

$$
\begin{aligned}
& k \lambda \frac{\partial^{3} u}{\partial y^{3}}+\frac{\partial^{2} u}{\partial y^{2}}+\lambda \frac{\partial u}{\partial y}+M u=-(G r \theta+G m C) \\
& \frac{1}{\operatorname{Pr}} \frac{\partial^{2} \theta}{\partial y^{2}}+\lambda \frac{\partial \theta}{\partial y}-\frac{1}{\operatorname{Pr}}(R+Q)=-D u \frac{\partial^{2} C}{\partial y^{2}}
\end{aligned}
$$


$\frac{1}{S c} \frac{\partial^{2} C}{\partial y^{2}}+\lambda \frac{\partial C}{\partial y}-K r C=0$

Relevant to the boundary conditions

$u=1, \theta=1, \quad C=1 \quad y=0$

$u \rightarrow 0, \theta \rightarrow 0, C \rightarrow 0 \quad y \rightarrow \infty$

\section{METHOD OF SOLUTION}

The solutions of the equations (8) and (9) are given by

$\theta=D_{1} e^{m_{2} y}+D_{2} e^{m_{4} y}$

$C=e^{m_{2} y}$

To solve the equation (7) we use the perturbation scheme considering the visco-elastic parameter $\mathrm{k}$ as the perturbation parameter as $k<<1$ in this problem for small shear rate.

Let us assume that the solution of the equation (7) subject to the boundary condition is of the form

$$
f(y)=f_{0}(y)+k f_{1}(y)+0\left(k^{2}\right)
$$

Substituting this in the equation (7) and by equating the coefficients of like terms of $\mathrm{k}$ and neglecting the higher order terms we get,

Zeroth order equation

$u_{0}^{\prime \prime}+\lambda u_{0}^{\prime}-M u_{0}=-G r \theta-G r C$

First order equation

$u_{1}^{\prime \prime}+\lambda u_{1}^{\prime}-M u_{1}=\lambda u_{0}^{\prime \prime \prime}$

Corresponding to boundary conditions

$y=0 \quad: \quad u_{0}=0, u_{1}=0$

$y \rightarrow \infty: u_{0}=0, u_{1}=0$

The solutions of the equations (12) and (13) are given by

$u_{0}=A_{1} e^{m_{2} y}+A_{2} e^{m_{4} y}+A_{3} e^{m_{2} y}+A_{4} e^{m_{6} y}$

$u_{1}=A_{5} e^{m_{6} y}+A_{6} e^{m_{2} y}+A_{7} e^{m_{4} y}+A_{8} e^{m_{2} y}+A_{9} e^{m_{8} y}$

And thus the velocity profile is given by

$$
u=A_{1} e^{m_{2} y}+A_{2} e^{m_{4} y}+A_{3} e^{m_{2} y}+A_{4} e^{m_{6} y}+k\left[A_{5} e^{m_{6} y}+A_{6} e^{m_{2} y}+A_{7} e^{m_{4} y}+A_{8} e^{m_{2} y}+A_{9} e^{m_{8} y}\right]
$$

\section{Skin - friction:}

The skin-friction coefficient at the plate is given by

$$
\frac{\tau^{\prime}}{\rho U^{2}} \tau=\left(\frac{\partial u}{\partial y}+k \lambda \frac{\partial^{2} u}{\partial y^{2}}\right)_{y=0}=m_{2} A_{1}+m_{4} A_{2}+m_{2} A_{3}+m_{6} A_{4}+k\left[m_{6} A_{5}+m_{2} A_{6}+m_{4} A_{7}+m_{2} A_{8}+m_{8} A_{9}\right]
$$

\section{Heat Transfer:}

The rate of heat transfer in terms of Nusselt number at the plate is given by 
$N u=\left(\frac{\partial \theta}{\partial y}\right)_{y=0}=m_{2} D_{1}+m_{4} D_{2}$

The mass flux at the wall in terms of Sherwood number is given by

$N u=\left(\frac{\partial C}{\partial y}\right)_{y=0}=m_{2}$

\section{RESULTS AND DISCUSSION}

A problem of steady two dimensional free convective hydro-magnetic flow of a viscoelastic fluid past a vertical porous surface in presence of radiation, chemical reaction of first order and Dufour effect has been analyzed in this paper. The viscoelastic effect is exhibited through the non-dimensional parameter $k$. Figures (1) to (6) characterize the velocity profile for various values of visco-elastic parameter along with a combination of other flow parameters present in the problem. The Prandtl number is considered as greater than $1($ i.e. $\operatorname{Pr}>1)$. It physically interprets the higher viscosity of governing fluid flow. The figures enable that the speed of the fluid enhanced in the neighbourhood of the plate but it will slow down as it goes away from the plate in case of externally cooled plate $(G r>0)$. This physical phenomenon of higher viscosity in the neighbourhood of the plate is noticed in both Newtonian fluid $(k=0)$ and non-Newtonian fluid $(k=0.1,0.2)$. The increasing values of viscoelasticity accelerate the speed of non-Newtonian fluid flow in comparison with the Newtonian fluid flow for externally cooled plate but a reverse behaviour is noticed during the fluid flows past an externally heated plate $(G r<0)$.
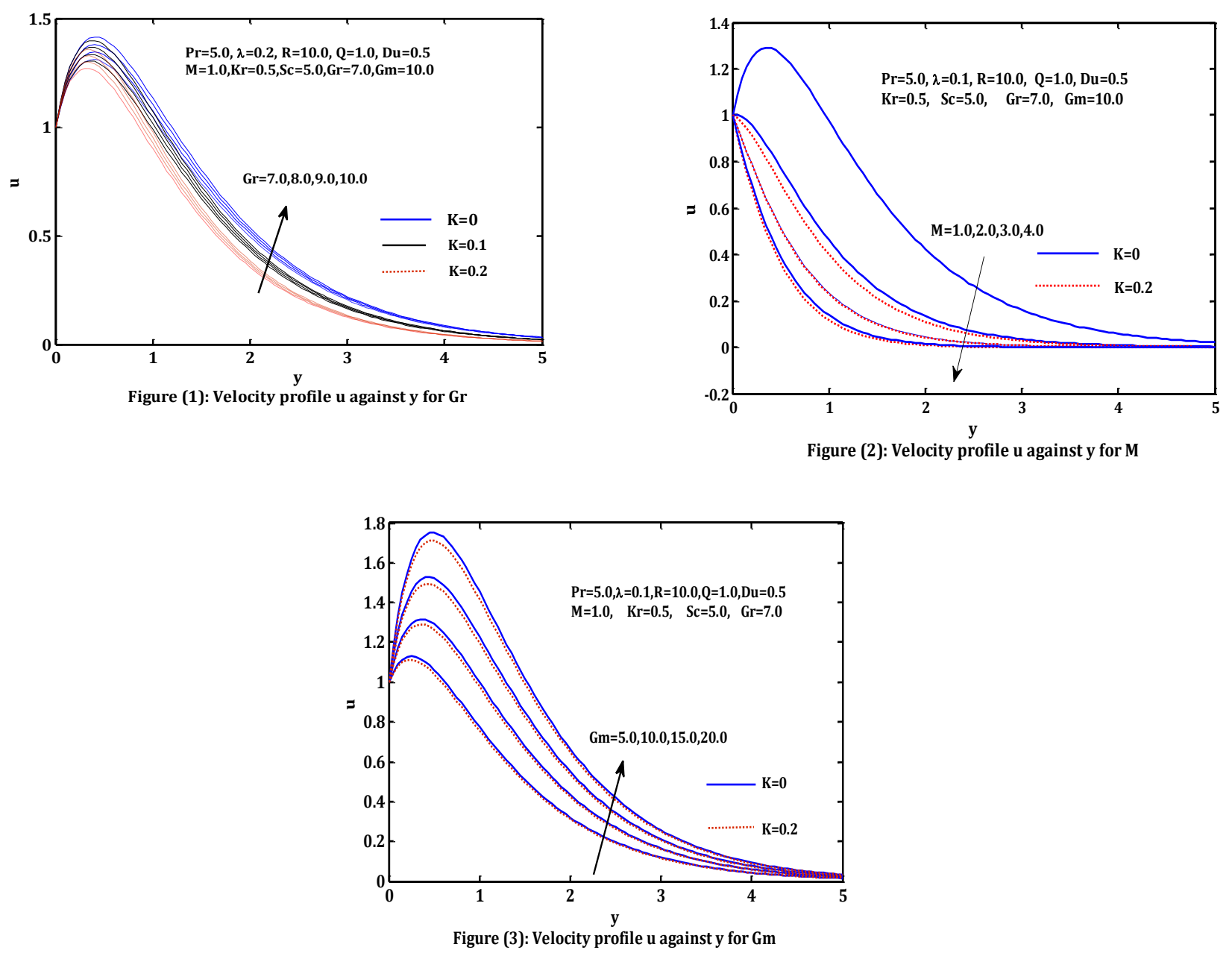

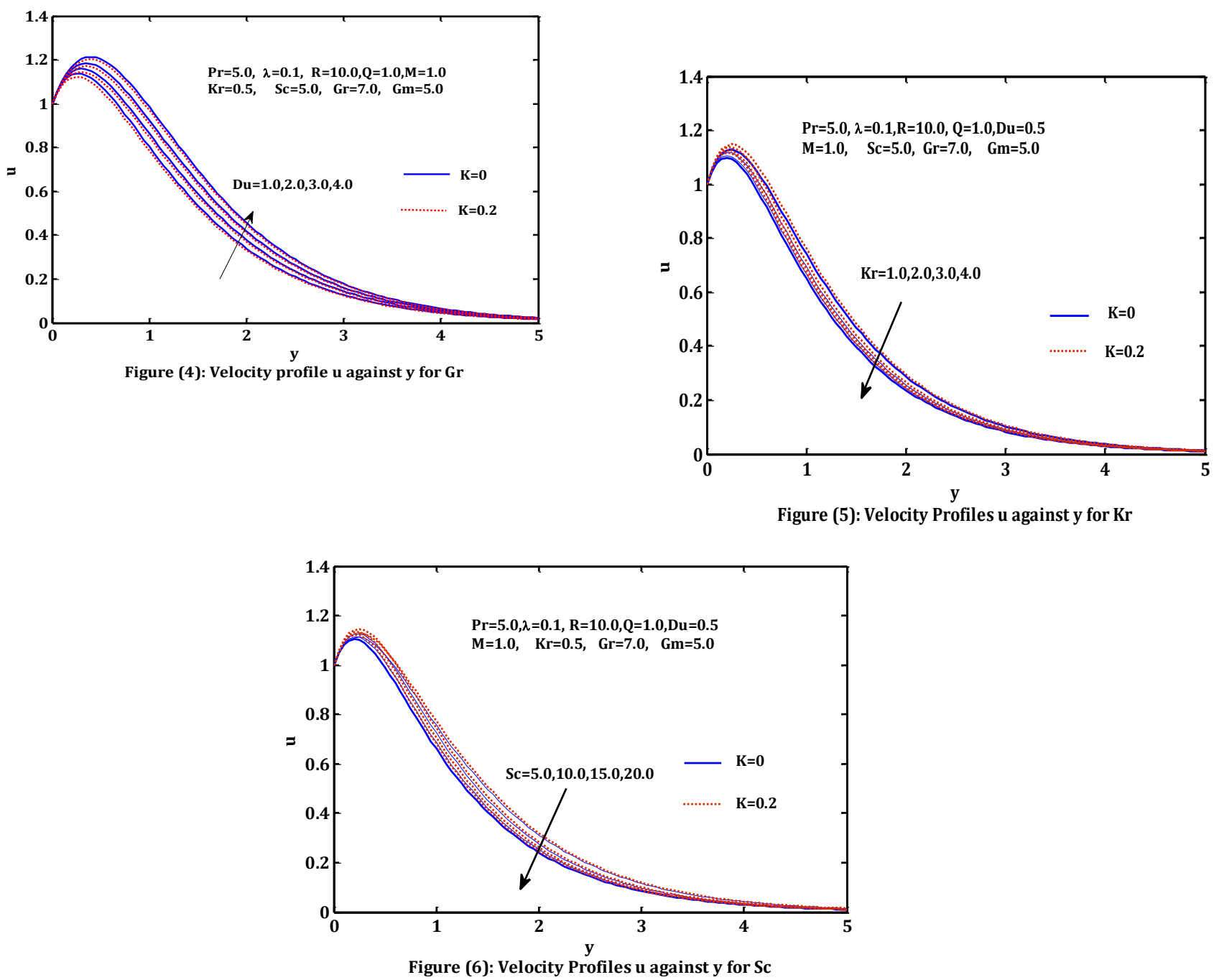

In figure (1), we have considered positive as well as negative values of thermal Grashof number. $(G r>0)$ Signifies that the fluid flow past an externally cooled plate and its negative value indicates that the flow is through an externally heated plate. The differences in flow patterns in both positive and negative values of Grashof number are noticed in the figure (1). In case of positive value of Grashof number $(G r)$, the velocity profile experiences a parabolic profile but for the flow past a heated plate a back flow in noticed for $(K \leq 0.1)$ as the fluid moves away from the plate. Figure (2) reveals the effect of Hartmann number $(M)$ on the velocity of the various fluid flow mechanisms. The application of transverse magnetic field creates a Lorentz force which has a retarding effect on the speed of the electrically conducting fluid flow. This diminishing behaviour is noticed in both Newtonian as well as non-Newtonian fluid flows for positive values of thermal Grashof number. Effects of Grashof number $(\mathrm{Gm})$ for mass transfer are analyzed graphically in figure (3) respectively. Increasing values of mass Grashof number $(\mathrm{Gm})$ raises the thickness of the fluid which in turn reduces the speed of both Newtonian and non-Newtonian fluid in combination of other flow parameters. Grashof number studies the nature of free convection and the effect of free convection on mass transfer is characterized through the nondimensional parameter $G m$. For the flow past a cooled plate, the increasing values of Grashof number modifies the speed of viscoelastic fluid flow and Newtonian fluid flows. The Dufour effect $(D u)$ is energy flux due to mass concentration gradient. It is exhibited through the Dufour effect parameter $(D u)$. The enhancement of Dufour number raises the speed of various fluid flows along with the increasing values of viscoelastic parameter shown in figure (4). Figure (5) illustrates the variation of the velocity distribution across the boundary layer for various values chemical 
reaction parameter $(K r)$. It is clear that the velocity of the fluid flow decreases with increase in the chemical reaction parameter. Effects of Schmidt number are analyzed graphically in figure (6) respectively. Increasing values of Schmidt number raises the thickness of the fluid which in turn reduces the speed of both Newtonian and non-Newtonian fluid in combination of other flow parameters. From practical point of view, the importance of shearing stress or viscous drag cannot be neglected. In this discussion, mainly we have concentrated the formation of shearing stress at externally cooled surface. Figure (7) and (8) seems temperature profiles for various values of $D u$ and $\lambda$; it shows that an increasing values of $D u$ the results is decreases and the reverse effect observed in $\lambda$. Figure (9) shows the variation of the temperature profiles for different values of the Prandtl number $(\operatorname{Pr})$. The result shows that an increase of the $\operatorname{Pr}$ results in a decrease in the thermal boundary layer thickness and a uniform temperature distribution across the boundary layer. The reason is that the smaller values of $\operatorname{Pr}$ are equivalent to increasing the thermal conductivities and therefore heat is able to diffuse away from the heated surface more rapidly than for larger values of $\operatorname{Pr}$. Hence the thicker the boundary layer is, the slower the rate of heat transfer is. The formulation of the effects of heat source $(Q)$ has been performed in the figure (10). This enables us to carry out the increasing $Q$ the distribution of the temperature across the boundary layer is decreases. Variation of temperature profiles for different values of thermal radiation parameter $(R)$ is shown in figure (11). The results show that the temperature profiles decreases with increase in the thermal radiation parameter and hence there would be an increase of thermal boundary layer thickness. Figure (11) indicates that there is a decrease in temperature as time increases. The concentration profiles for different values of chemical reaction parameter $(K r)$ and

$\lambda$ is shown in figure (12) and (13). There is a fall in the concentration due to the increasing values of the chemical reaction parameter. This shows that the diffusion rates can be tremendously altered by chemical reaction; and the same effect observed in figure (13) with $\lambda$. Figure (14) shows the concentration profiles for various values of Schmidt number $(S c)$. It is observed that an increase in Sc leads to a decrease in the concentration distribution, because the smaller values of Sc are equivalent to an increase in the chemical molecular diffusivity. Figure (15) and (16) represent the behaviour of shearing stress and Nusselt number experienced by the motion of various values of Grasoff number and Radiation parameters of fluid flow mechanisms against Prandtl number respectively. It is observed that in both cases, increasing values of Grasoff number and Radiation parameters lead to fall the magnitude of viscous drag along with the increasing values of Grasoff number and Radiation parameters.

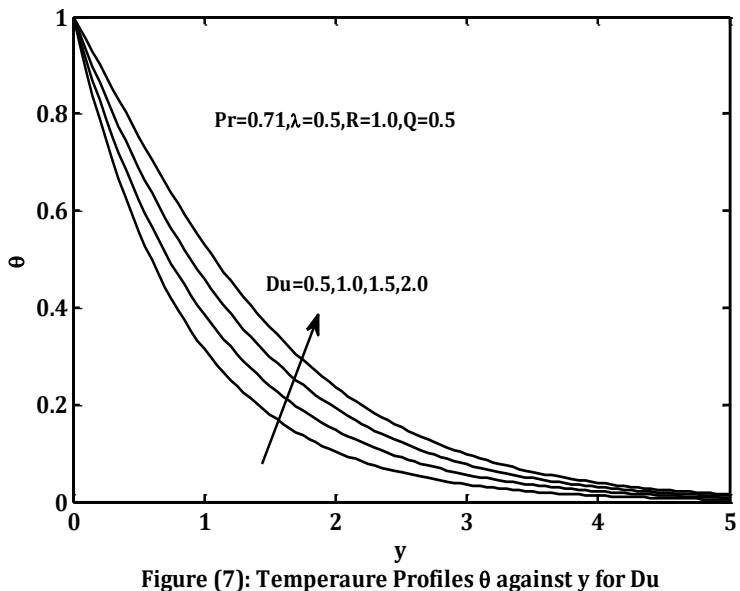

Figure (7): Temperaure Profiles $\theta$ against y for Du

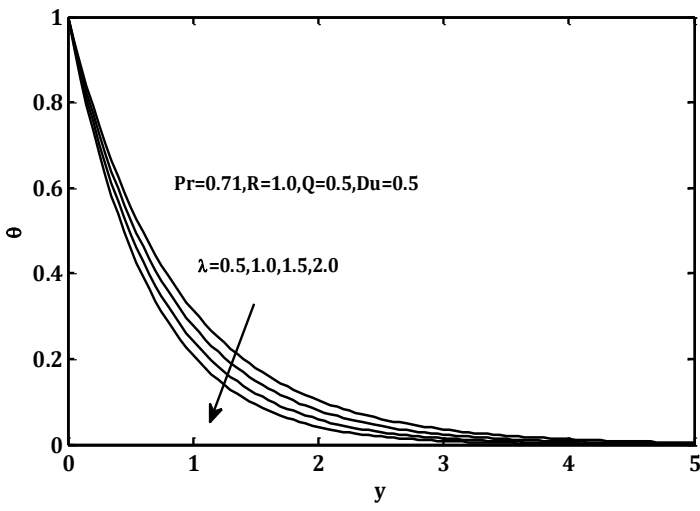

Figure (8): Temperaure Profiles $\theta$ against $y$ for $\lambda$ 


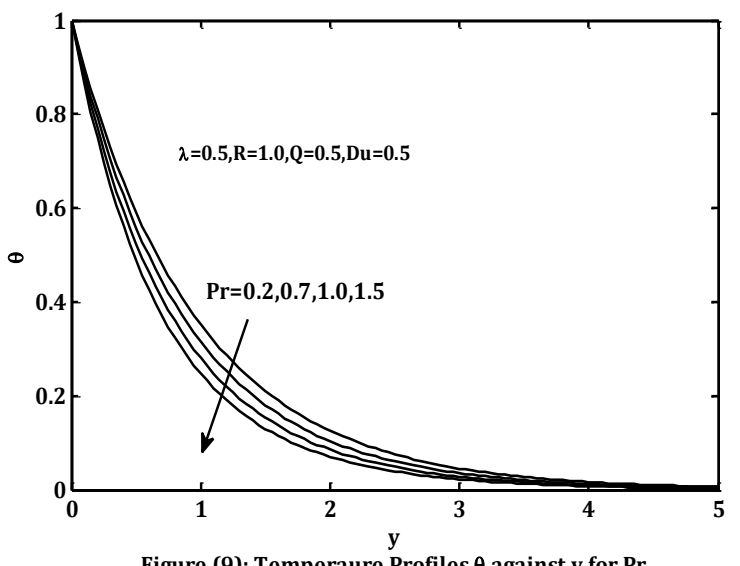

Figure (9): Temperaure Profiles $\theta$ against $y$ for $\operatorname{Pr}$

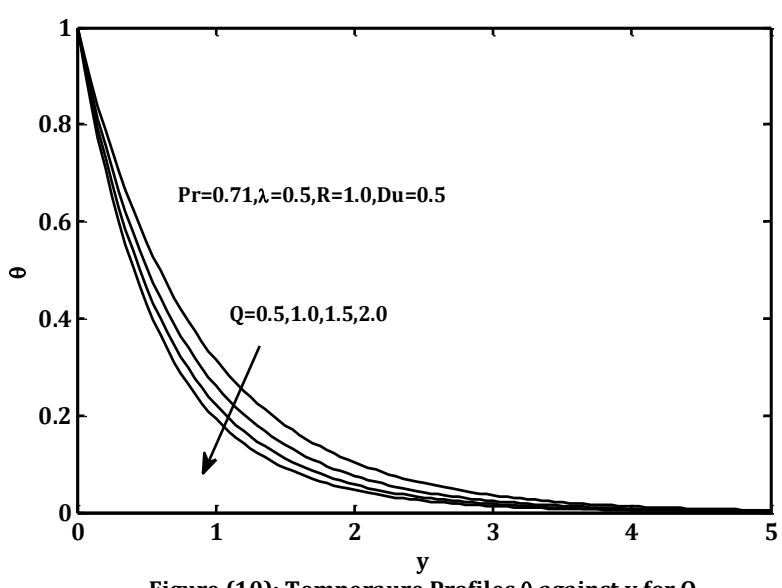

Figure (10): Temperaure Profiles $\theta$ against y for $Q$

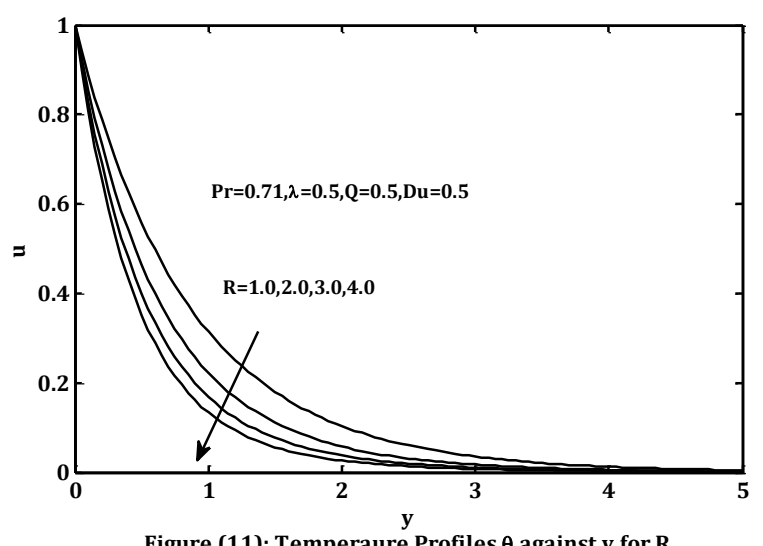

Figure (11): Temperaure Profiles $\theta$ against $y$ for $R$
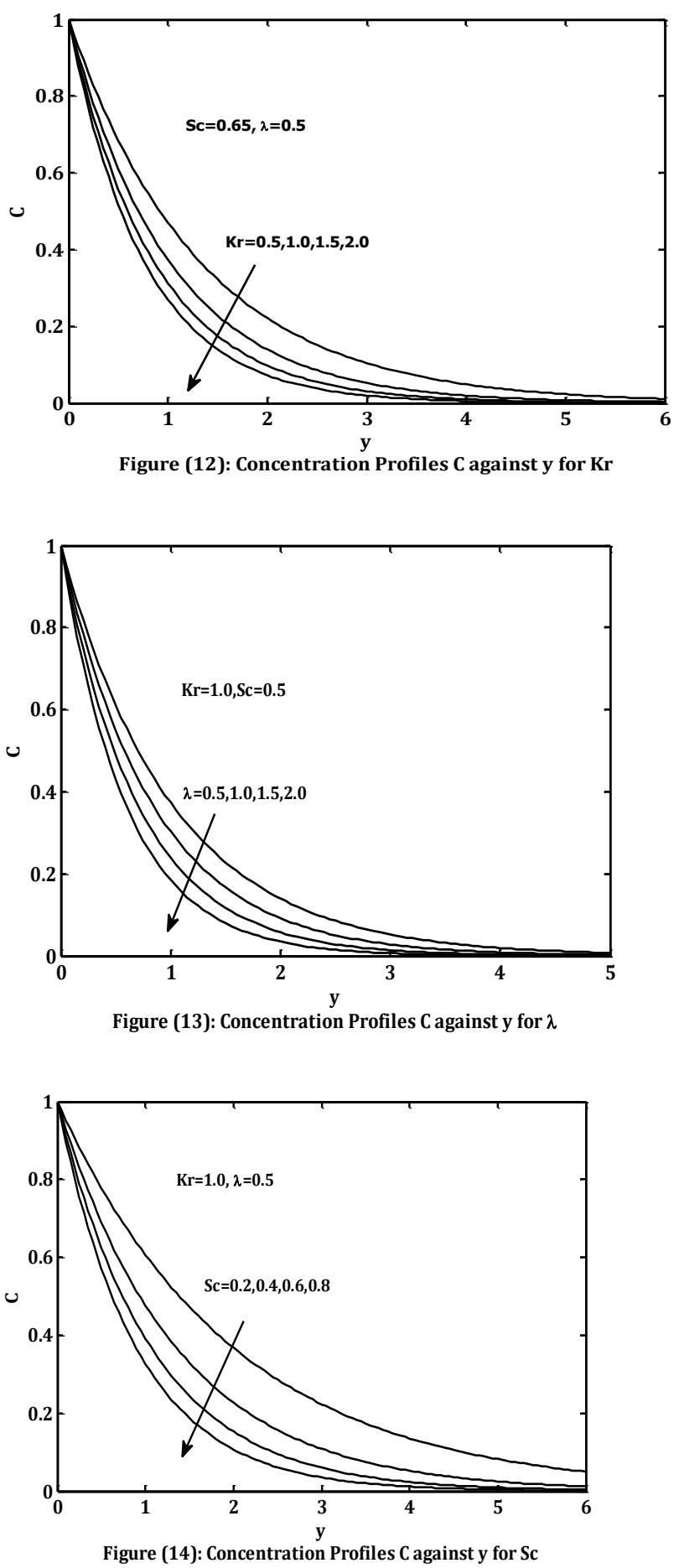


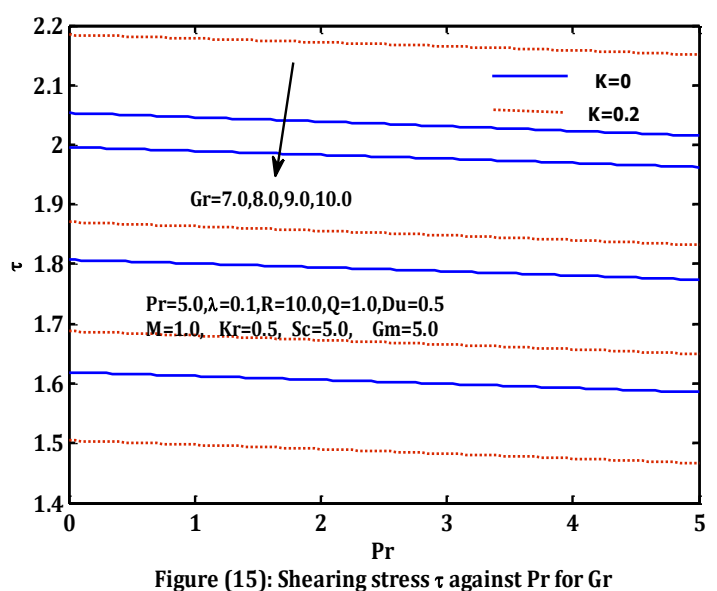

Figure (15): Shearing stress $\tau$ against $\operatorname{Pr}$ for $G r$

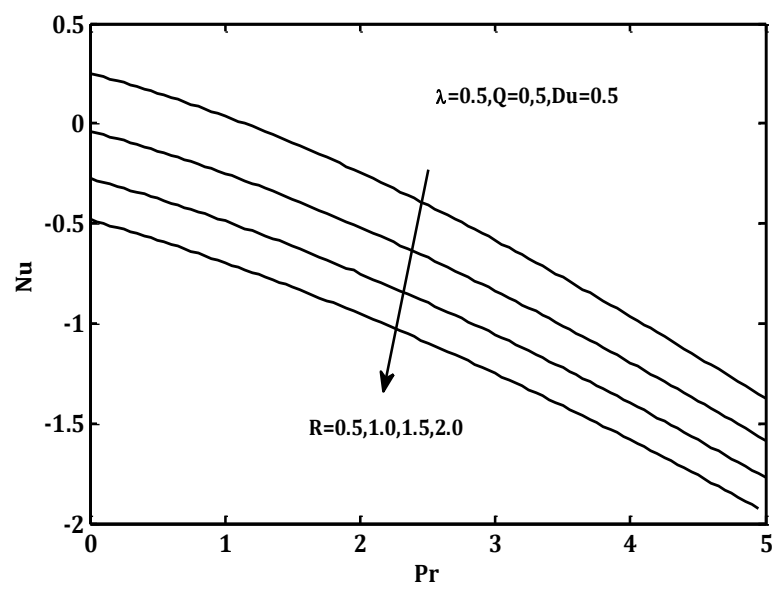

Figure (16): Nusselt number against Pr for $R$

\section{CONCLUSIONS}

An analysis of a homogeneous first order chemical reaction between the fluid and species concentration to study diffusion thermo (Dufour effect) and radiation effects on steady MHD free convection flow of a viscoelastic, electrically conducting ,radiating fluid past an infinite vertical plate in the presence of transverse applied magnetic field is performed. This study leads to following conclusions.

- The velocity field is considerably affected by the viscoelastic parameter at all points of the fluid flow region in combination of other physical parameters.

- For externally cooled plate, the velocity profile accelerates but the reverse pattern is observed for externally heated plate in both Newtonian and non-Newtonian fluids.

- The effects of magnetic parameter, Grashof number for mass transfer and Schmidt number are significant in the velocity field and the behaviours of the profiles are noticed similar in all the cases for externally cooled plate.

- The speed of the velocity profile increases with the enhancement of the Dufour number in Newtonian and nonNewtonian cases.

- The enhancement of Prandtl number and Dufour number reveals the rising trend of shearing stress but the reverse tendency is observed for externally cooled surface when Schmidt number increases in Newtonian and non- Newtonian fluid flow mechanism.

- For externally cooled surface, the chemical reaction parameter subdues the magnitude of shearing stress in both Newtonian and non-Newtonian fluid flow phenomenon.

- The temperature and the mass concentration fields are not significantly affected by the viscoelastic parameter in the fluid flow region.

\section{REFERENCES}

[1] Chen, C H. On the analytic solution of MHD flow and heat transfer for two types of viscoelastic fluid over a stretching sheet with energy dissipation. Int. J. Heat Mass Transfer, 53, 2010, pp. 4264-4273

[2] Dulal Pal and Hiranmoy Mondal. Soret and Dufour effects on MHD non-Darcian mixed convection heat and mass transfer over a stretching sheet with non-uniform heat source/sink , Physica B, 407, 2012, pp. 642-651

[3] Dulal Pal and Sewli Chatterjee (2014): MHD mixed convective heat and mass transfer in a visco elastic fluid in a porous medium towards a stretching sheet with viscous-ohmic heating and chemical reaction, The Canadian journal of chemical engineering, Vol. 92, 2011

[4] Andersson, H I. Acta Mech., 95, 1992, p. 227.

[5] Vajravelu, $\mathrm{K}$ and Roper, T. Flow and heat transfer in a second grade fluid over a stretching sheet, International Journal Non-Linear Mech., 34, 1999, pp. 1031-1036.

[6] Rajagopal, K R and Gupta, A S. An exact solution for the flow of a non-Newtonian fluid past an infinite porous plate, Meccanica, 19, 1984, pp. 158-160.

[7] Rajagopal, K R. On boundary conditions for fluids of the differential type, in: A. Sequeira (Ed.), Navier Stokes Equations and Related non- Newtonian Fluid.

[8] Rajagopal, K R. On the creeping flow of second order fluid, J. Non-Newtonian Fluid Mech. 15, 1994, pp. 239246.

[9] Linear Problems, Plenum Press, New York, 1995, pp. 273-278.

[10] Abel, M S, Khan, S K and Prasad, K V. Int. J. Numer. Methods Heat Fluid Flow, 11, 2011, p. 779. 
[11] Char, M I. Heat and mass transfer in a hydromagnetic flow of the viscoelastic fluid over a stretching sheet, $J$. Math. Anal. Appl. 186, 1994, pp. 674-689.

[12] Sarma, M S and Rao, B N. Heat transfer in a viscoelastic fluid over a stretching sheet, J. Math. Anal. Appl. 222, 1998, pp. 268-275.

[13] Makinde, O D. Int. J. Numer. Methods Heat Fluid Flow, 19, 2009, p. 546.

[14] Makinde, O D and Charles. W M. Appl. Comput. Math.-Appl. Comput. Math, 9, 2010, p. 243.

[15] Sibanda, P and Makinde, O D. (2010): Int. J. Numer. Methods Heat Fluid Flow, 20, p. 269.

[16] Cortell, R. A note on flow and heat transfer of a viscoelastic fluid over a stretching sheet, Internat. J. NonLinear Mech., 41, 2005, pp. 78-85.

[17] Rafael Cortell Bataller. Effects of heat source/sink, radiation and work done by deformation on flow and heat transfer of a viscoelastic fluid over a stretching sheet, Computers and Mathematics with Applications, 53, 2007, pp. 305-316

[18] Rita Choudhury and Sajal Kumar Das. Visco-Elastic MHD fluid flow over a vertical plate with Dufour and Soret effects, International Journal of Scientific \& Engineering Research, Vol. 4 (7), 2013, pp. 11-17

[19] Abel, S, Joshi, M and Sonth, R M. Heat transfer in MHD visco-elastic fluid over a stretching surface, Z. Angew. Math. Mech. 81, 2001, pp. 691-698.

[20] Abel, S and Veena P H. Viscoelastic fluid flow and heat transfer in a porous medium over a stretching sheet, Internat. J. Non-Linear Mech. 33, 1998, pp. 531-540.

[21] Chenna Kesavaiah, D, Satyanarayana, P V, Sudhakaraiah, A and Venkataramana, S. Natural convection heat transfer oscillatory flow of an elastico-viscous fluid from vertical plate, IJRET, Vol. 2 (6), 2013, pp. 959-966

[22] Ch Kesavaiah, D, Satyanarayana, P V and Venkataramana, S. Effects of the chemical reaction and radiation absorption on an unsteady MHD convective heat and mass transfer flow past a semi-infinite vertical permeable moving plate embedded in a porous medium with heat source and suction, Int. J. of Appl. Math and Mech. Vol. 7 (1), 2011, pp. 52-69

[23] Bhavana, M and Chenna Kesavaiah, D. Perturbation solution for thermal diffusion and chemical reaction effects on MHD flow in vertical surface with heat generation, International Journal of Future Revolution in Computer Science \& Communication Engineering, Vol. 4 (1), 2018, pp. 215-220

[24] Bhavana, M, Chenna Kesavaiah, D and Sudhakaraiah, A. The Soret effect on free convective unsteady MHD flow over a vertical plate with heat source, International Journal of Innovative Research in Science, Engineering and Technology, Vol. 2 (5), 2013, pp. 1617-1628

[25] Srinathuni Lavanya, Chenna Kesavaiah, D and Sudhakaraiah, A. Radiation, heat and mass transfer effects on magnetohydrodynamic unsteady free convective Walter's memory flow past a vertical plate with chemical reaction through a porous medium, International Journal of Physics and Mathematical Sciences, Vol. 4 (3), 2014, pp. 57-70

[26] Mallikarjuna Reddy, B, Chenna Kesavaiah, D and Ramana Reddy, G V. Effects of radiation and thermal diffusion on MHD heat transfer flow of a dusty viscoelastic fluid between two moving parallel plates, $A R P N$ Journal of Engineering and Applied Science, Vol. 13 (22), 2018, pp. 8863 


\section{APPENDIX}

$$
\begin{aligned}
& m_{2}=-\left(\frac{S c \lambda+\sqrt{S c^{2} \lambda^{2}+4 K r S c}}{2}\right), m_{4}=-\left(\frac{\lambda \operatorname{Pr}+\sqrt{\lambda^{2} \operatorname{Pr}^{2}+4(R+Q)}}{2}\right) \\
& m_{6}=-\left(\frac{\lambda+\sqrt{\lambda^{2}+4 M}}{2}\right), m_{8}=-\left(\frac{\lambda+\sqrt{\lambda^{2}+4 M}}{2}\right) \\
& D_{1}=-\frac{D u m_{2}^{2}}{m_{2}^{2}+\lambda m_{2}-(R+Q)}, D_{2}=-\left(1-D_{1}\right), A_{1}=-\frac{G r D_{1}}{m_{2}^{2}+\lambda m_{2}+M}, A_{2}=-\frac{G r D_{2}}{m_{4}^{2}+\lambda m_{4}+M} \\
& A_{3}=-\frac{G m}{m_{2}^{2}+m_{2}-B_{1}}, A_{4}=\left(1-A_{1}-A_{2}-A_{3}\right), A_{5}=\frac{\lambda m_{6}^{3} A_{4}}{m_{6}^{2}+\lambda m_{6}+M}, A_{6}=\frac{\lambda m_{2}^{3} A_{1}}{m_{2}^{2}+\lambda m_{2}+M} \\
& A_{7}=\frac{\lambda m_{4}^{3} A_{2}}{m_{4}^{2}+\lambda m_{4}+M}, A_{8}=\frac{\lambda m_{2}^{3} A_{3}}{m_{2}^{2}+\lambda m_{2}+M} A_{9}=-\left(A_{5}+A_{6}+A_{7}+A_{8}\right)
\end{aligned}
$$

